Review

\title{
Trends in the discovery of new drugs for Mycobacterium tuberculosis therapy with a glance at resistance
}

\author{
Vahid Lohrasbi ${ }^{\mathrm{a}}$, Malihe Talebi ${ }^{\mathrm{a}}$, Abed Zahedi Bialvaei ${ }^{\mathrm{a}}$, Lanfranco Fattorini ${ }^{\mathrm{b}}$, \\ Michel Drancourt ${ }^{\mathrm{c}, \mathrm{d}}$, Mohsen Heidary ${ }^{\mathrm{a}}$, Davood Darban-Sarokhalil ${ }^{\mathrm{a}, *}$ \\ ${ }^{a}$ Department of Microbiology, Faculty of Medicine, Iran University of Medical Sciences, Tehran, Iran \\ ${ }^{\mathrm{b}}$ Department of Infectious Diseases, Istituto Superiore di Sanità, Rome, Italy \\ ${ }^{\mathrm{c}}$ Institut Hospital-Universitaire (IHU) Mediterranée Infection, AP-HM, Marseille, France \\ d Aix-Marseille Université, Unité de Recherche sur les Maladies Infectieuses et Tropicales Emergentes (URMITE), UM63, CNRS 7278, IRD 198, INSERM 1095, Marseille, \\ France
}

\section{A R T I C L E I N F O}

\section{Keywords:}

Mycobacterium tuberculosis

Drug resistance

Mechanisms

New drugs

MDR

XDR

TDR

HDTs

\begin{abstract}
A B S T R A C T
Despite the low expensive and effective four-drug treatment regimen (isoniazid, rifampicin, pyrazinamide and ethambutol) was introduced 40 years ago, TB continues to cause considerable morbidity and mortality worldwide. In 2015, the WHO estimated a total of 10.4 million new tuberculosis (TB) cases worldwide. Currently, the increased number of multidrug-resistant (MDR-TB), extensively-drug resistant (XDR-TB) and in some recent reports, totally drug-resistant TB (TDR-TB) cases raises concerns about this disease. MDR-TB and XDR-TB have lower cure rates and higher mortality levels due to treatment problems. Novel drugs and regimens for all forms of ТВ have emerged in recent years. Moreover, scientific interest has recently increased in the field of hostdirected therapies (HDTs) in order to identify new treatments for MDR-TB. In this review, we offer an update on the discovery of new drugs for TB therapy with a glance at molecular mechanisms leading to drug resistance in Mycobacterium tuberculosis.
\end{abstract}

\section{Introduction}

Tuberculosis (TB) is one of the top 10 causes of death worldwide [1]. Until the late nineteenth century, it amounted to a death sentence for patients diagnosed with this disease. The discovery of Mycobacterium tuberculosis (MTB) as the causative agent by Robert Koch in 1882 paved the way for better treatments of TB [2]. Streptomycin (SM) (1943) and para-aminosalicylic acid (PAS) (1946) were among the first clinically important drugs to be developed [3]. Both showed activity against MTB and were rapidly followed by the use of isoniazid (INH) (1952), pyrazinamide (PZA) (1952), ethambutol (EMB) (1961) and rifampicin (RIF) (1966) among others. Therefore these two decades were called the golden age of TB antibiotics [3]. The introduction of these drugs for TB treatment immediately led to a sharp and continuous decline of ТВ incidence throughout the world. However, the disease suddenly came back in the 1980s in association with the rising epidemic of the acquired immune deficiency syndrome (AIDS) and the emergence of drug-resistant forms [4].

The emergence and diffusion of drug-resistant TB is now recognized as one of the most dangerous threats to global TB control [5]. Patients with drug-resistant TB, particularly multidrug-resistant (MDR; caused by MTB strains resistant to at least RIF and INH) or extensively drugresistant (XDR; caused by MDR MTB strains with additional resistance to at least one fluoroquinolone (FQ) and $1 \mathrm{~s}$-line injectable drug) need long, toxic, and expensive drug treatment with a highly unsatisfactory proportion of outcomes [6-8]. There is an urgent need for the development and more efficient evaluation of new TB drugs and shorter treatment regimens. The WHO's 2015 annual TB report states that "without new TB drugs and regimens, it will be very difficult to improve treatment outcomes in the near future" [4]. In this review, we give an update on the discovery of new drugs for TB therapy with a glance at molecular mechanisms leading to current and new drug resistance in MTB.

\section{Current drugs}

Current drugs are discussed in this part. Table 1 summarizes the commonly used TB drugs with their genetic basis and related information in resistance.

\footnotetext{
* Corresponding author. Department of Microbiology, Faculty of Medicine, Iran University of Medical Sciences, Tehran, Iran.

E-mail addresses: davood_darban@yahoo.com, darban.d@iums.ac.ir (D. Darban-Sarokhalil).
} 


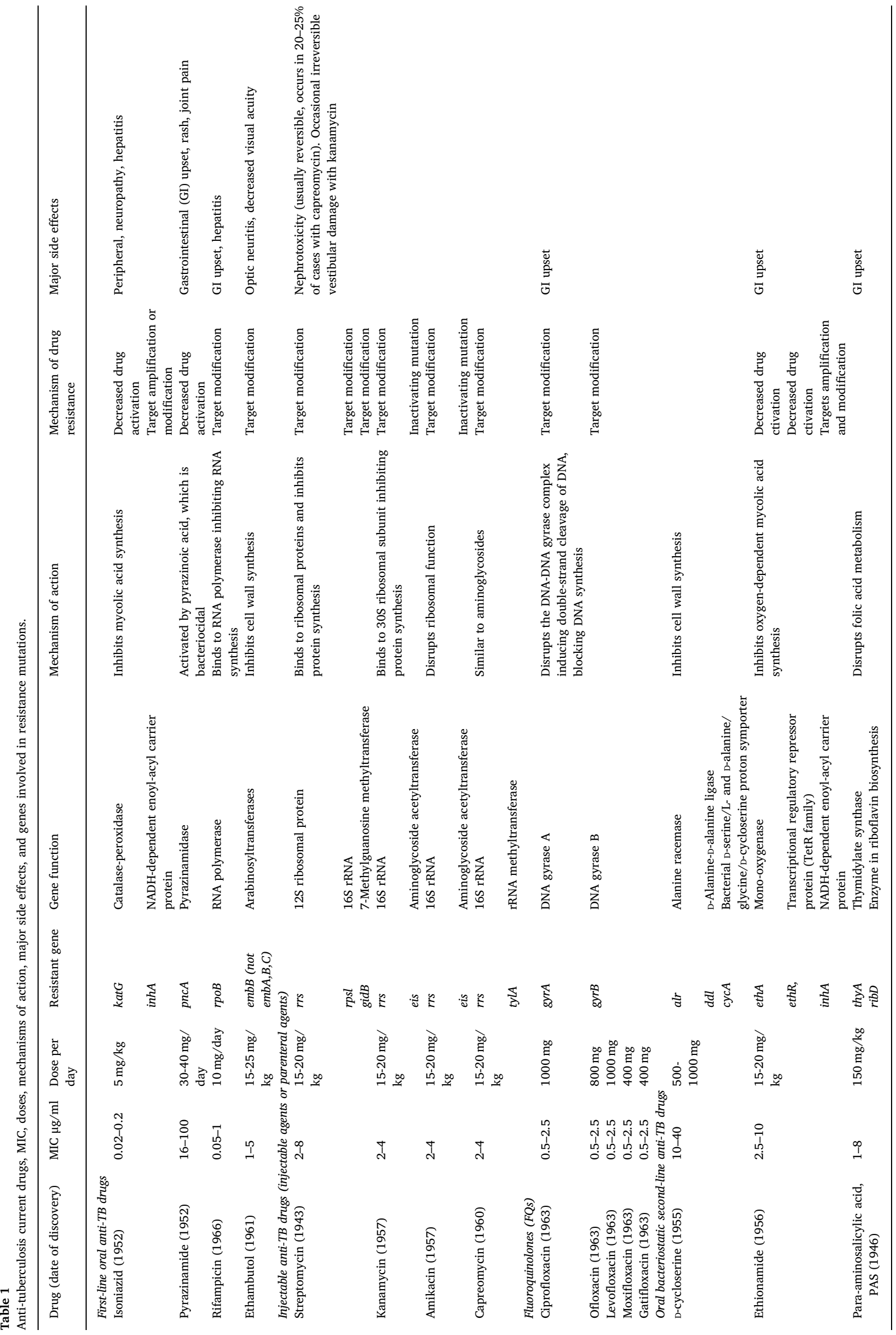




\subsection{First-line oral anti-TB drugs}

\subsubsection{Isoniazid}

Isoniazid (isonicotinic acid hydrazide) is a first-line pro-drug that requires activation by the catalase/peroxidase enzyme encoded by kat $G$ [9]. The active species (isonicotinic acyl radical or anion) reacts with nicotinamide adenine dinucleotide (NAD), forming the INH-NAD adduct, which then inhibits InhA causing inhibition of cell wall mycolic acid synthesis. INH is active only against growing MTB, but not against non-growing bacilli (9). On the other hand, INH tolerance in nongrowing organisms may be caused by the mycobacterial DNA-binding protein 1 (MDP1), a histone-like protein which down-regulates kat transcription, and could lead to tolerance to INH [10].

Resistance to INH is a complex process. Mutations in several genes, including $k a t G, a h p C$, inh $A$, $k a s A$ and $n d h$, have all been associated with INH resistance. Mutations in $k a t G$ are the major mechanism of INH resistance $[9,11,12]$. The $k a t G$ S315 mutation is the most common mutation in INH-resistant strains, accounting for 50-95\% of INH resistant clinical isolates $[9,13]$. In contrast to kat $G$ mutations, which usually cause high-level resistance, mutations in inhA or its promoter region are usually associated with low level resistance (minimum inhibitory concentrations [MICs] of $0.02-0.2 \mu \mathrm{g} / \mathrm{ml}$ ) and are less frequent than kat $G$ mutations (Table 1) [11,13]. Mutations in inhA not only cause INH resistance, they also confer cross-resistance to the structurally related drug ethionamide (ETH) [14]. Other genes including kasA, $a h p C, n d h$, and the $a h p C$-oxyR intragenic region have been associated with resistance to INH, but their impact on resistance among clinical isolates remains unclear [15-17].

\subsubsection{Rifampicin}

Rifampicin, which was introduced in 1966, is a lipophilic ansamycin also highly bactericidal and sterilizing for MTB. An important characteristic of RIF is that it is active against actively growing and slowly metabolizing (non-growing) bacilli [18]. Although RIF is known to interfere with RNA synthesis by binding to the $\beta$ subunit of the RNA polymerase, a recent study showed that RIF binding to the rpoB target induces hydroxyl radical formation in susceptible, but not resistant bacilli, and may contribute to the killing effect of RIF [19]. Mutations in a 'hot-spot' region of the 81-bp region of $r p o B$ are found in about $96 \%$ of RIF-resistant MTB isolates [20]. Mutations at positions 531, 526 and 516 are the most frequent mutations in RIF-resistant strains. Mutations in $r р о B$ generally result in cross-resistance to all rifamycins, including rifabutin (RBT), but some RIF-resistant strains are RBT-susceptible [21].

\subsubsection{Ethambutol}

Like PZA, EMB interferes with the biosynthesis of cell wall arabinogalactan [22]. Arabinosyl transferase, encoded by embB, an enzyme involved in the synthesis of arabinogalactan, is the target of EMB in MTB [23]. Mutations in embCAB operon, in particular embB, and occasionally $e m b C$, are responsible for resistance to EMB [23]. The embB codon 306 mutation is most frequent in clinical isolates resistant to EMB, accounting for as much as $68 \%$ of resistant strains $[24,25]$. While mutations on EmbB306 lead to certain amino acid changes and caused EMB resistance, other amino acid substitutions had little effect on EMB resistance [26]. However, about 35\% of EMB-resistant strains (MIC of $1-5 \mu \mathrm{g} / \mathrm{ml}$ ) did not have $e m b B$ mutations, suggesting other mechanisms of resistance [27]. Mutations in ubiA, encoding the decaprenylphosphoryl-5-phosphoribose (Dppr) synthase involved in cell-wall synthesis, have recently been found to cause higher-level EMB resistance in conjunction with $e m b B$ mutations [28].

\subsubsection{Pyrazinamide}

Pyrazinamide is a paradoxical drug with unique sterilizing activity in anti-TB treatment $[29,30]$. In striking contrast to common antibiotics that kill or inhibit growing bacteria, PZA inhibits non-growing bacteria and has activity against growing bacteria under acidic $\mathrm{pH}$ only [31]. One key characteristic of PZA is its ability to inhibit semidormant bacilli residing in acidic environments [18]. PZA is a pro-drug that has to be converted to its active form, pyrazinoic acid (POA), in order for the pyrazinamidase/nicotinamidase enzyme encoded by the pncA gene of MTB to become active [32]. Pyrazinamide has multiple effects on MTB, including interference with membrane energy production, and inhibition of RpsA (ribosomal protein S1), which is involved in translation, and PanD, which is involved in pantothenate and co-enzyme A synthesis [29,31,33,34].

Mutations in the pncA gene are the main mechanism of PZA resistance in MTB [32,35]. Most PZA-resistant MTB strains (72-99\%, average 85\%) have mutations in pncA, but some do not [35-39].

RpsA target mutations are usually associated with low-level PZA resistance (MICs of $16-100 \mu \mathrm{g} / \mathrm{ml}$ ). More recently, panD was found to be involved in PZA resistance [29]. panD mutations were identified in naturally PZA-resistant Mycobacterium canettii strains and some PZAresistant MDR-TB strains. $M$. canettii, a member of the MTB complex, is intrinsically resistant to PZA, and has mutations in both rpsA48 and panD [40].

\subsection{Parenteral anti-TB drugs}

\subsubsection{Aminoglycosides (streptomycin, kanamycin/amikacin) and capreomycin}

Streptomycin is an aminocyclitol glycoside antibiotic that was first used in the treatment of TB. Streptomycin inhibits protein synthesis by binding to the $30 \mathrm{~S}$ subunit of the bacterial ribosome, causing misreading of the mRNA message. Resistance to SM is caused by mutations in the S12 protein encoded by the rpsL gene and 16S rRNA encoded by the rrs gene [41]. Mutations in rpsL and rrs are the principal mechanism of SM resistance, accounting for about 50\% and $20 \%$ of SM-resistant strains, respectively $[41,42]$. Nonetheless, about $\sim 30 \%$ of SM-resistant strains with low-level resistance (MICs of $2-8 \mu \mathrm{g} / \mathrm{ml}$ ) do not have any mutations in $r p s L$ or $r r s$, and may have mutations in gidB encoding a conserved 7-methylguanosine (m(7)G) methyltransferase specific for 16S rRNA [43]. Mutations at the rrs position 1400 cause high-level resistance to kanamycin (KM) and amikacin (AK) [44]. SM-resistant strains usually remain susceptible to KM and AK. Mutations in the promoter region of the eis gene, encoding aminoglycoside acetyltransferase, cause low-level resistance to KM, but not to AK [45].

Mutations in tlyA encoding rRNA methyltransferase and the 23S rRNA gene rrs (A1401G and G1484T) are involved in chlorpheniramine resistance [46]. Mutants with A1401G in the rrs gene could cause resistance to KM and chlorpheniramine [47]. Multiple mutations may occur in the rrs gene in one strain, conferring cross-resistance among these agents [47].

\subsubsection{Fluoroquinolones (ofloxacin, moxifloxacin, levofloxacin, and gatifloxacin)}

Fluoroquinolones (FQ) belong to the quinolone class of antibiotics inhibiting bacterial DNA gyrase and topoisomerase IV, enzymes required for vital processes such as replication, transcription, recombination, and chromosomal supercoiling [48]. The main cause of FQ-resistance (FQ-R) is the disruption of the binding site for the drug in the DNA-gyrase enzyme (encoded by the gyrA and gyrB genes) [49]. A completely different mechanism proposed to explain FQ-R involves the Pentapeptide Repeat Protein (PRP) family [50]. Pentapeptide proteins are composed of tandemly repeated amino acid sequences with a consensus sequence of $[\mathrm{S}, \mathrm{T}, \mathrm{A}, \mathrm{V}][\mathrm{D}, \mathrm{N}][\mathrm{L}, \mathrm{F}]-[\mathrm{S}, \mathrm{T}, \mathrm{R}][\mathrm{G}]$. The three-dimensional structure of the first member of the PRP family was determined for the fluoroquinolone resistance protein (MfpA) from MTB [51]. The mycobacterial pentapeptide protein MfpA would protect DNA gyrase from FQ activity by: i) Overexpression and binding of MfpA to the GyrA subunit of DNA gyrase would inhibit FQ DNA binding, ii) thus blocking the formation of the GyrA-FQ complex [52]. However, in contrast to the 


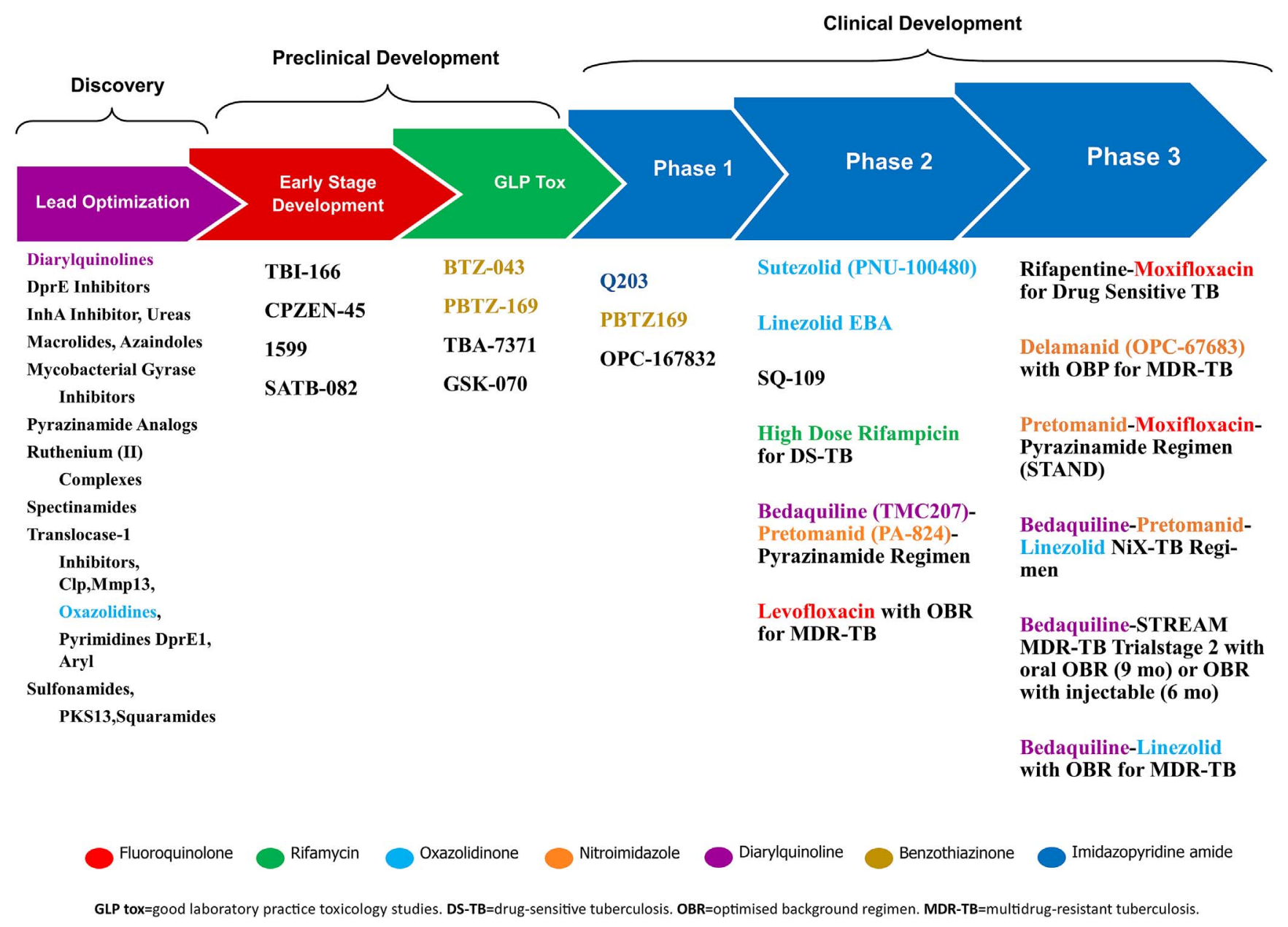

Fig. 1. Research and development pipeline for new anti-TB drugs.

observations made on Escherichia coli, where pentapeptide proteins protect the DNA gyrase from FQ, MfpA in MTB was found to enhance the inhibitory activity of FQs on the topoisomerase [53].

On the whole, codons 94, 90 and 88 of gyrA conferred high-level FQ resistance, as did multiple mutations [54,55]. On the other hand, the much less common gyrB mutations (up to a total of 10-15\%) were generally, but not consistently, associated with lower levels of FQ-R; however, combined gyrA and gyrB mutations could result in a much higher level of resistance [56,57]. A recent study in FQ-monoresistant clinical isolates of MTB revealed high levels of efflux pump pstB transcripts in a few of these isolates, suggesting a contribution of the pump to resistance [58].

\subsection{Oral bacteriostatic second-line anti-TB drugs}

\subsubsection{Ethionamide/prothionamide}

Ethionamide is a derivative of isonicotinic acid, and is a bactericidal agent active only against MTB. The MICs of ETH for MTB are 0.5-2 $\mu \mathrm{g}$ / $\mathrm{ml}$ in liquid media, $2.5-10 \mu \mathrm{g} / \mathrm{ml}$ in $7 \mathrm{H} 11$ agar and $5-20 \mu \mathrm{g} / \mathrm{ml}$ in Lowenstein-Jensen medium. Like INH, ETH is also a prodrug that is activated by EtaA/EthA (a mono-oxygenase) and inhibits the same target of INH, i.e. InhA, of the mycolic acid synthesis pathway [59,60]. The structure and activity of prothionamide are almost identical to those of ETH. EthA is an FAD-containing enzyme that oxidises ETH to the corresponding S-oxide, which is further oxidised to 2-ethyl-4-amidopyridine, presumably via the unstable oxidised sulfinic acid intermediate [61]. EthA also activates thioacetazone, thiocarlide, thiobenzamide and perhaps other thioamide drugs, which explains the cross-resistance between these agents (e.g., isoxyl). In addition, mutations in the InhA target confer resistance to both ETH and INH [61].

\subsection{2. $D$-cycloserine/terizidone}

D-cycloserine (DCS) is a bacteriostatic agent used in the treatment of MDR-TB. Terizidone is a combination of two molecules of DCS. The MICs of DCS for MTB ranged from 10 to $40 \mu \mathrm{g} / \mathrm{ml}$ depending on the culture media used. DCS is an analogue of D-alanine that inhibits the synthesis of peptidoglycan by blocking the action of D-alanine:D-alanine ligase (Ddl). It also inhibits D-alanine racemase (Alr) involved in the interconversion of L-alanine and D-alanine, which then serves as a substrate for Ddl [62]. Overexpression of alrA encoding D-alanine racemase causes resistance to DCS in Mycobacterium smegmatis [63,64]. Although the cellular target(s) of D-cycloserine in MTB is still unknown, a study suggests that the primary target of DCS in MTB is Ddl [65]. It was reported that $c y c A$ encoding $\mathrm{D}$-serine, $\mathrm{L}$ - and $\mathrm{D}$-alanine and glycine transporter involved in the uptake of D-cycloserine was defective in $M$. bovis BCG, which could be related to its natural resistance to DCS [66].

\subsubsection{Para-aminosalicylic acid}

Para-aminosalicylic acid (PAS) is a bacteriostatic agent with MICs of $1-8 \mu \mathrm{g} / \mathrm{ml}$ for MTB [11]. Interference with the folic acid biosynthesis and inhibition of iron uptake have been proposed as two possible mechanisms of action [67,68]. Para-aminosalicylic acid was recently shown to be a prodrug that inhibits folic acid metabolism by incorporation into the folate pathway through activation by dihydropteroate synthase (DHPS, encoded by FolP) and dihydrofolate synthase (DHFS, encoded by FolC). It can generate a toxic 
hydroxyldihydrofolate antimetabolite, which inhibits dihydrofolate reductase (DHFR, encoded by $d f r A$ [Rv2763c]) [69,70]. The mechanism of PAS resistance is not well understood. Mutations in thy A encoding thymidylate synthase, which reduce the utilization of tetrahydrofolate, were responsible for resistance in about $37 \%$ of PAS-resistant clinical isolates [71]. Mutations in folC and $d f r A$ have also been found in PASresistant strains [71,72]. Overexpression of the PAS drug-activating enzyme DHFS (FolC) restored sensitivity to PAS in the resistant strain, while overexpression of the target DHFR caused PAS resistance [54,72]. More studies are needed to validate the PAS resistance genes identified in clinical isolates.

\section{New anti-TB drugs}

After five decades of near inactivity in TB drug development, the past years have seen the emergence of a promising TB drug pipeline (Fig. 1) [73]. Combining these new drugs with existing TB drugs offers hope for regimens that are better tolerated, shorter in duration and with fewer drug-drug interactions when compared with existing regimens. The current anti-TB drug pipeline shows four drugs in clinical trial at phase II or III for MDR-TB treatment. Bedaquiline (BDQ) and delamanid (DLM) are in trial phase III; their approval in several countries was justified by the emergency situation caused by MDR-TB. Sutezolid (SZD) and pretomanid (PMD), two other new drug candidates, are in trial phases II and III, respectively (Table 2) [74]. In this section we will give some information on these drugs and other two (Q203 and PBTZ169) in phase I studies, the mechanisms of action of the six drugs are shown in Fig. 2.

\subsection{Bedaquiline}

After a gap of more than 50 years without new TB drugs, the U.S. Food and Drug Administration (FDA) approved BDQ in 2012 for the treatment of MDR-TB [75]. Bedaquiline, (formerly known as TMC207 and R207910) is a diarylquinoline drug. It was discovered in a high throughput phenotypic screening for compounds active against the saprophytic mycobacteria, M. smegmatis, and subsequently showed to demonstrate activity against $M$. bovis BCG and MTB [76].

Bedaquiline kills both dormant and actively replicating mycobacteria by inhibiting mycobacterial adenosine triphosphate (ATP) synthase, an essential membrane-bound enzyme, interfering with energy production and disrupting intracellular metabolism [76]. ATP synthase is a critical enzyme in the ATP synthesis of MTB. Binding of BDQ to the oligomeric and proteolipid subunit-c of the mycobacterial ATP synthase leads to inhibition of ATP synthesis, which subsequently results in bacterial death [77]. The gene encoding the subunit-c of the ATP synthase is denoted as atpE and its amino-acid sequence is highly conserved in non-related MTB isolates [78]. AtpE forms a part of the F1F0 proton ATP synthase, a transmembrane protein complex that generates ATP from proton translocation, and inhibition of ATP biosynthesis has been shown to kill both actively dividing and nondividing bacteria [79]. Bedaquiline does not interfere with mammalian ATP synthase activity. Human mitochondrial ATP synthase sensitivity to BDQ was 20,000 times lower than its sensitivity to the mycobacterial enzyme [80].

Ineffective or incomplete treatment can select resistant and mutant MTB strain. Insight into the functional target of BDQ first emerged with the generation of resistant mutants followed by whole-genome resequencing. The studies identified mutations in the atpE gene encoding the membrane-bound subunit-c of F0ATP synthase, which were independently confirmed through comparative sequence analyses of naturally BDQ-susceptible and -resistant mycobacteria, and selected BDQ-resistant MTB strains [81,82]. Bedaquiline-resistant mutants arose at a rate of one in $10^{8}$ organisms [76]. In vitro resistance to BDQ in mycobacteria has been shown to involve mutations in atpE [83]. However, resistant mutants that did not have any mutations in atpE or in the other genes encoding components of ATP synthase have been identified, suggesting alternative mechanisms of resistance or perhaps other targets for this drug [82]. Mutations in the transcriptional regulator Rv0678, leading to upregulation of efflux pump MmpL5, were recently found to cause cross-resistance involving both clofazimine (CFZ) and BDQ [84,85].

Bedaquiline has been investigated in phase IIb studies for the treatment of MDR-TB. In the two-stage phase II trial that provided evidence of BDQ's safety and efficacy, the investigators enrolled patients with positive sputum smears and sensitivity to at least three of the five classes of drugs used in the background anti-mycobacterial drug regimen for MDR pulmonary TB [86]. In the first stage, 47 patients were randomly assigned in double-blind fashion, to receive 8 weeks of placebo or BDQ in addition to the background anti-mycobacterial drug regimen. Upon completion of the 8-week trial period, the rate of sputum-culture conversion among BDQ-treated patients (48\%) was markedly higher than of patients who received placebo (9\%). In the second stage, patients were randomly assigned, also in double-blind fashion, to receive placebo or BDQ for 24 weeks, both in combination with their background anti-mycobacterial drug regimen, for a total of approximately 18-24 months. There were 79 patients in the BDQ group and 81 in the placebo group. In the second stage, the median time to sputum-culture conversion was significantly shorter in the BDQ group than in the placebo group ( 83 days vs. 125 days; $\mathrm{P}<.001$ ). These two trials thus demonstrated BDQ's effectiveness on the basis of sputumculture conversion.

Phase III trial of BDQ, currently underway, is planned to confirm its efficacy from earlier phase II clinical trials and to obtain additional safety data. Bedaquiline will get traditional FDA approval if results of phase III trial confirm that the drug actually provides clinical benefit [87]. The main concerns among members of the FDA regarding the safety of BDQ is the unexplained higher number of deaths in the BDQ arm of the randomized control trial [88], since the commonest adverse reaction observed is the QTc interval increase in the electrocardiogram (ECG) [88-90]. About $11.4 \%$ of patients taking BDQ died during clinical trials compared with $2.5 \%$ of those taking placebos. As the drug carries some significant risks, it is mandated to be used only in patients who do not have other treatment options [77]. Bedaquiline carries a socalled black box warning for patients and healthcare professionals that it can affect the heart's electrical activity, causing prolongation of the QT interval, which could lead to an abnormal and potentially fatal heart rhythm. Accordingly, the FDA has approved BDQ as part of a combination therapy to treat adults with MDR pulmonary TB when other alternatives are not available. The FDA also granted fast-track designation, priority review and orphan-product designation to BDQ [77].

Bedaquiline is highly active against MTB. It demonstrated similar activity against clinical isolates resistant to first-line drugs, including INH, RIF, PZA, EMB and SM, and against moxifloxacin [76]. In addition, BDQ showed similar activity against a number of MDR strains [76]. The MICs of MDR-TB strains, including an XDR MTB strain and a MDR strain also resistant to injectables and FQs, ranged from 0.06 to $0.12 \mu \mathrm{g} / \mathrm{ml}$ [81]. The WHO and the CDC published provisional recommendations for the appropriate use of BDQ in the treatment of MDR-TB in 2013 [91]. Bedaquiline should be reserved for patients with MDR-TB when an effective regimen with PZA and $4 \mathrm{~s}$-line drugs, as recommended by the WHO, cannot otherwise be designed. It is also recommended for the treatment of MDR-TB with documented resistance to any FQ. The recommended dose is $400 \mathrm{mg}$ daily for 2 weeks, followed by $200 \mathrm{mg}$ three times per week during 22 weeks. It should be administered with food to maximize absorption. The WHO recommended that it should not be given for more than 6 months, whereas the CDC suggested that treatment for more than 24 weeks could be considered on a case-by-case basis when an effective regimen cannot otherwise be provided [92]. 


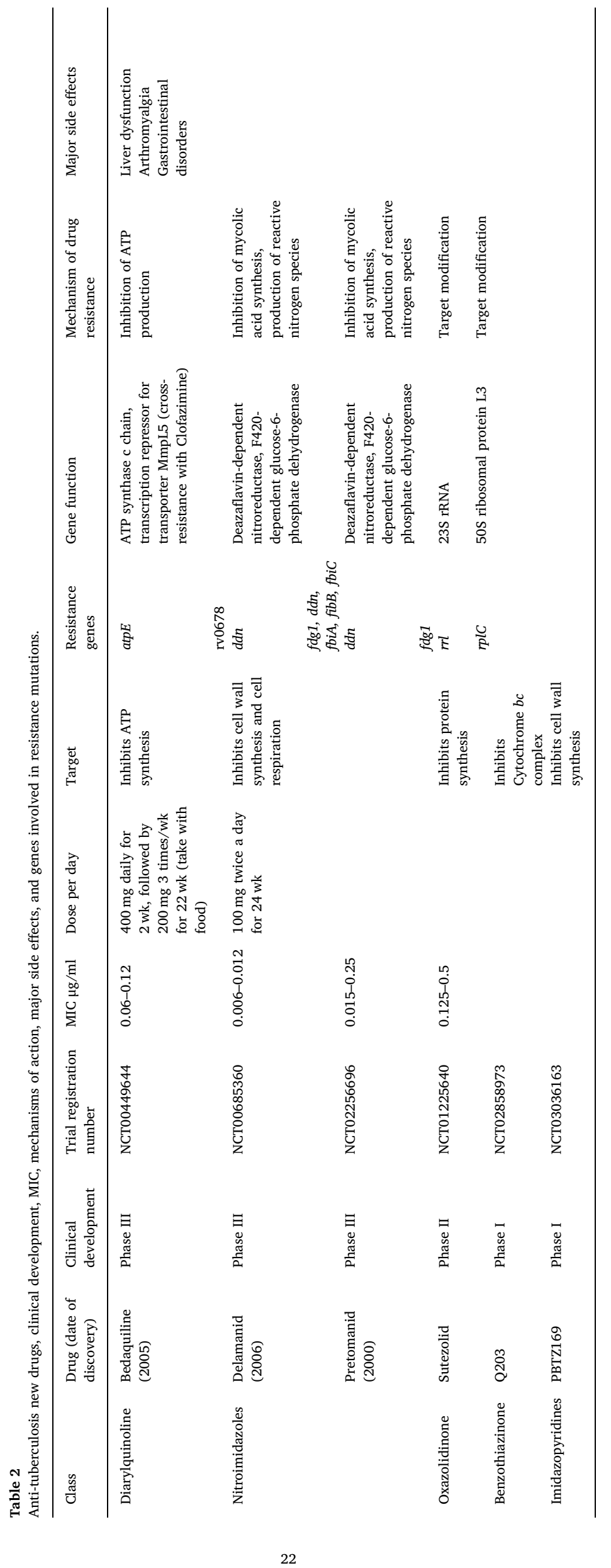




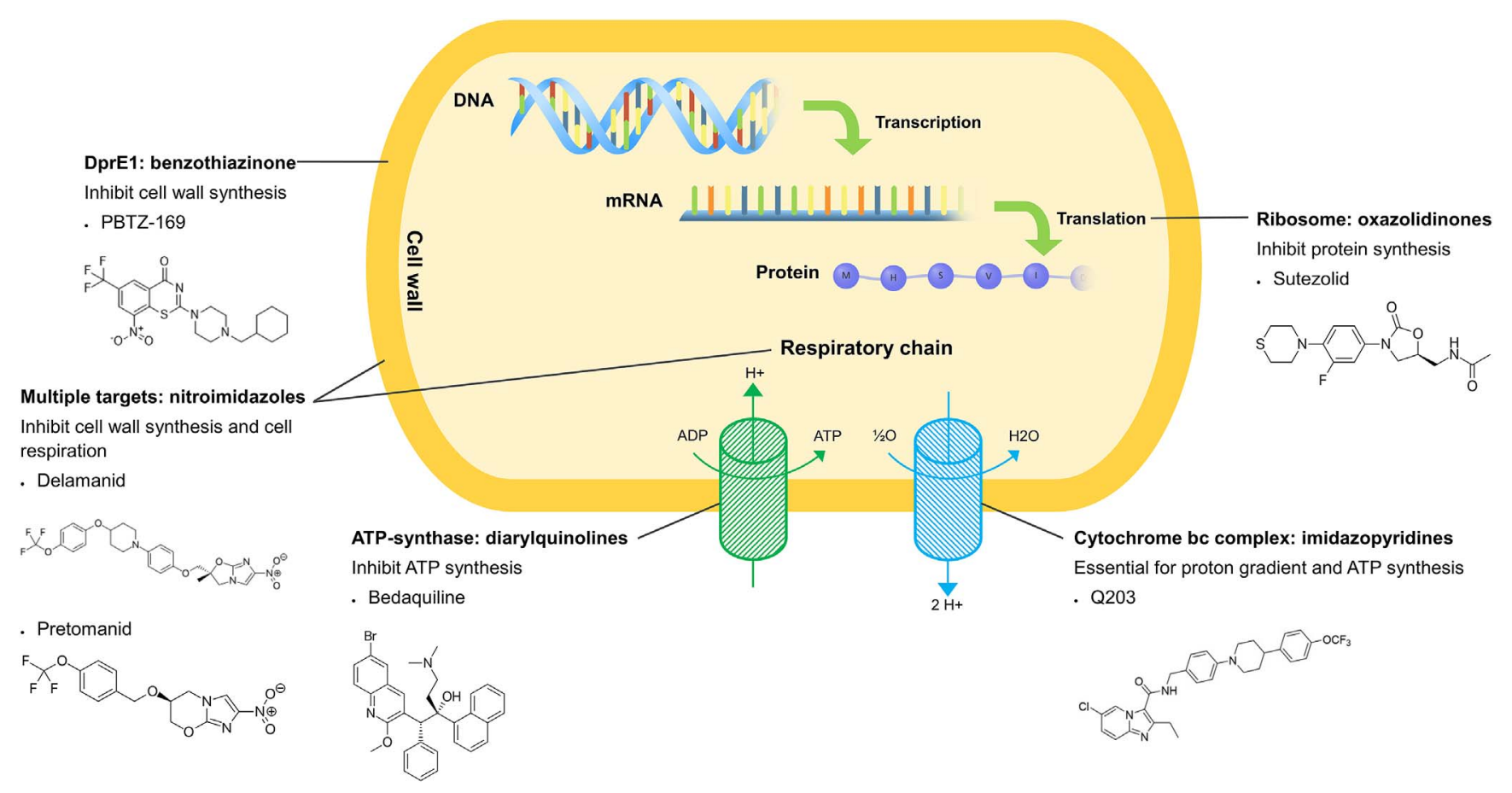

Fig. 2. Mechanisms of action of new anti-TB drugs.

\subsection{Pretomanid}

Pretomanid (formerly known as PA-824), a nitroimidazole, is a potent inhibitor of both actively replicating bacteria and nonreplicating MTB (MICs of $0.015-0.25 \mu \mathrm{g} / \mathrm{ml}$ ). Activity against the hypoxic non-replicating population is particularly important for the development of new TB drugs. The PMD was initially thought to inhibit cell-wall lipid biosynthesis [93]. It is a prodrug activated by a deazaflavin-dependent nitroreductase (Ddn) (Rv3547) to form three primary metabolites, the main one being des-nitroimidazole (des-nitro) [94,95]. Des-nitro compounds generate reactive nitrogen species, including nitric oxide (NO), and are responsible for the anaerobic activity of these compounds and may synergize the mycobacterial killing with the host-derived NO produced by macrophages [95].

Mutations in $d d n$ encoding the deazaflavin-dependent nitroreductase and in fgd1 (Rv0407) encoding the F420-dependent glucose-6phosphate dehydrogenase, both of which are involved in F420 coenzyme biosynthesis, are found in mutants resistant to PMD and complementation restored susceptibility [95]. Mutations in fgd1 and $d d n$ found in 65 MTB complex strains representing the main phylogenetic lineages did not appear to cause resistance to PMD (MIC of $0.25 \mu \mathrm{g} / \mathrm{ml}$ ) [96]. Interestingly, $M$. canetti, a member of the MTB complex, was found to be intrinsically resistant to PMD, with an MIC of $8 \mu \mathrm{g} / \mathrm{ml}$ [96]; however, the basis of its resistance is unknown. The PMD-MFX-PZA regimen that is currently in Phase III trial for shortening antiTB treatment [97].

\subsection{Delamanid}

Delamanid (formerly known as OPC-67683) is a new drug that has recently received approval from the European Union and Japan for the treatment of MDR-TB in combination with other drugs [98,99]. It belongs to the class of nitroimidazoles and has shown potent in vitro and in vivo activity against both drug-susceptible and drug-resistant strains of MTB in preclinical and clinical development [100].

Delamanid acts by inhibiting the synthesis of mycobacterial cell wall components, methoxy mycolic acid and ketomycolic acids of MTB [101]. Delamanid has activity against non-replicating persister bacteria
[102]. Similar to PMD, it is a prodrug that is activated by the deazaflavin-dependent nitroreductase enzyme (Ddn). A reactive intermediate metabolite, formed between delamanid and a desnitro-imidazooxazole derivative, is considered to play a vital role in the inhibition of mycolic acid production [103]. Because of DLM bactericidal and sterilizing activity, it can also be considered as a core drug [101,104]. It does not show cross-resistance with other anti-TB drugs [101,104] and it is effective in increasing both bacteriological conversion and treatment outcomes, and in reducing mortality [101,105].

Mutations in one of the five coenzyme F420 genes ( $f g d 1, d d n, f b i A$, $f b i B$ and $f b i C$ ) are associated with resistance to DLM [3]. Mutations of $d d n$ and/or $f g d 1$ have already been reported to be associated with resistances to DLM and PMD, a closely related nitroimidazole-oxazine drug, respectively $[96,98]$. Likewise, mycobacterial strains with mutated $f b i A$ or $f b i B$ were shown to lack coenzyme F420 and to be resistant to PMD [106]. FbiA (homolog synonym cofD) is highly conserved among archaea and bacteria that are known to produce F420. The most likely variant conferring DLM resistance was the mutation FbiA D49Y [107]. In vitro frequencies of spontaneous DLM resistance are comparable to those of INH, that is, ranging from $1 / 10^{6}$ to $1 / 10^{5}$ MTB organisms [4].

In phase II clinical trials, the drug was used in combination with standard treatments, such as four or five drugs including EMB, INH, PZA, RIF, aminoglycoside antibiotics, and FQs. Healing rates (measured as sputum culture conversion) were significantly better in patients who additionally took DLM [108]. The main results of clinical trials came from a phase IIB trial together with studies of safety and efficacy. In July 2012, a phase IIB trial including DLM plus a background regimen resulted in more study subjects becoming non-infectious after two months than the trial including a placebo plus a background regimen. The trial was conducted in 17 centers in nine countries [108]. The follow-up stage of a Phase III trial of the safety and efficiency of DLM as an addition to an optimized background regimen (OBR) for the treatment of MDR-TB in adults was recently completed [109]. It is anticipated that results will be published in 2018. The use of DLM in addition to OBR for treatment of MDR-TB in children is being investigated in Phase I and II trials. Partial results were presented in 2015 [109,110]. The most common side effects are nausea, vomiting and dizziness 
[111]. These may affect as many as a third of all patients. There is also a serious side effect called QT prolongation. QT prolongation is an alteration of the electrical activity of the heart [111]. It can cause a lifethreatening abnormality of the heart rhythm. Anxiety, pins \& needles, and shaking are other important side effects [112]. No other serious treatment emergent adverse effects have been observed in the clinical trials [111,112].

In a subsequent assessment of the 14-day early bactericidal activity of the compound against MTB-infected patients in South Africa, DLM administered at doses of 200 and $300 \mathrm{mg}$ daily resulted in a decrease in the sputum MTB burden. These findings were similar to those on the potent anti-TB drug RIF in previous studies of its early bactericidal activity $[113,114]$. DLM has MICs of $0.006-0.024 \mu \mathrm{g} / \mathrm{ml}$, and thus appears to be 20 times more active than PMD. It is available in $50 \mathrm{mg}$ tablets and the recommended dose is two tablets taken twice a day with food. It needs to be taken for six months. The European Medicines Agency has issued a conditional marketing authorization for DLM (Deltyba, $50 \mathrm{mg}$ tablets). It should be used as a part of an appropriate combination regimen for pulmonary MDR-TB in adult patients in whom the current approved regimen cannot be used because of resistance or intolerability.

\subsection{Sutezolid}

Oxazolidinones are originally a class of antibiotics approved for the treatment of drug-resistant Gram-positive bacterial infections [115]. Oxazolidinones have significant activity against MTB, with MICs of $0.125-0.5 \mu \mathrm{g} / \mathrm{ml}$ [116]. The oxazolidinones inhibit an early step of protein synthesis by binding to ribosomal $50 \mathrm{~S}$ subunits, most likely within domain $\mathrm{V}$ of the 23S rRNA peptidyl transferase, and forming a secondary interaction with the $30 \mathrm{~S}$ subunit [117]. However, in the case of linezolid (LZD), the only currently licensed oxazolidinone, serious hematologic and neuro-logic toxicities arise during long-term use due to the inhibition of mitochondrial protein synthesis that often requires dose reduction or discontinuation [118,119].

Mutations at G2061T and G2576T in the 23S rRNA of MTB can cause high levels of resistance to LZD, with MICs of $32 \mu \mathrm{g} / \mathrm{ml}$ and $16 \mu \mathrm{g} / \mathrm{ml}$, respectively, but mutants with a low level of resistance (MICs of $4-8 \mu \mathrm{g} / \mathrm{ml}$ ) have no mutations in 23S rRNA [120]. Mutations in the $r p l C$ encoding ribosomal protein L3 (T460C mutation) were putatively involved in LZD resistance in MTB [121], largely accounting for phenotypes with low level of resistance [122]. A recent study from China suggests that resistance to LZD was already seen in about $11 \%$ of MDRTB strains; however, only $30 \%$ of these strains had mutations in the $23 \mathrm{~S}$ rRNA gene or the rplC gene [122], suggesting other, but currently unknown possible mechanisms.

Sutezolid (formerly known as PNU-100480) is an oxazolidinone and an analogue of LZD, with preliminary evidence of superior efficacy against MTB compared to LZD both in vitro and in a mouse model of TB $[116,123,124]$. Sutezolid was safe and well-tolerated in Phase I and II clinical studies and demonstrated potential efficacy in the treatment of TB. Phase I studies in healthy volunteers by administration of SZD ( $600 \mathrm{mg}$ twice daily for 28 days) revealed neither abnormal hematologic or biochemical findings nor instances of peripheral or ophthalmic neuropathy [125]. These data potentially indicate a safety profile superior to the similarly dosed LZD. In vivo studies in the chronic mouse model of TB demonstrated that the addition of SZD to the standard TB regimen has the potential to significantly shorten the treatment. It not only reduced the numbers of bacteria in the lungs more quickly, but also led to a relapse-free cure with shorter treatment duration $[125,126]$. A Phase II trial demonstrated that SZD had significant early bactericidal activity and may have clinical efficacy in humans in a larger Phase II trial [127]. Bedaquiline is apparently the best drug to pair with SZD in a new TB regimen for all forms of drug susceptible and drug resistant TB [128].

Sutezolid received Orphan Drug designation in both U.S. and E.U. and is currently in clinical development for the treatment of adult pulmonary TB caused by drug-sensitive or drug-resistant strains of MTB. Sutezolid was safe and well-tolerated at doses up to $1200 \mathrm{mg}$ daily for up to 14 days, or $600 \mathrm{mg}$ twice daily for up to 28 days [125].

\subsection{Q203}

Q203, an imidazopyridine anti-tubercular compound that also acts on the respiratory chain (Fig. 2), just entered Phase I clinical trials (Fig. 1) [4]. Q203 was discovered after medicinal chemistry optimization and a high-throughput screening (HTS) performed against infected human macrophages [129]. Q203 targets the cytochrome b subunit (QcrB) of the cytochrome $b c 1$ complex. This complex is an essential component of the respiratory electron transport chain of ATP synthesis. Q203 causes a rapid depletion of intracellular ATP at an IC50 of $1.1 \mathrm{nM}$ and interrupts ATP homeostasis in dormant MTB at an IC50 of $10 \mathrm{nM}$. Both of these values are better than BDQ's measures, and they explain Q203's excellent killing profile in chronic MTB infection models [130].

The Phase I clinical trial (clinicaltrials.gov identifier: NCT02530710) enrolled healthy patients is a dose-escalation study starting at a dose of $100 \mathrm{mg}$ that will be adjusted based on PK analysis. Further combination trials will be needed to determine whether Q203 can act synergistically in combination with other second-line agents to treat MDR-TB, which are no longer susceptible to INH or RIF [130].

\subsection{PBTZ169}

A new series of piperazine-containing benzothiazinones (PBTZ) has shown highly potent activity against drug-susceptible and drug-resistant MTB. PBTZ169 is compatible with all TB drugs and appears to have synergies with BDQ and CFZ. A Phase I trial of PBTZ169 was completed in the Russian Federation in July 2016, and a second Phase I trial will be undertaken in Switzerland in 2017. A Phase IIa trial is expected to start towards the end of 2016 in the Russian Federation [4].

\section{Totally drug-resistant TB}

Some recent reports use the term totally drug-resistant TB (TDR-TB) to describe TB caused by MTB strains that are resistant to all available first-line and second-line TB drugs [131]. The presence of TDR-TB was first observed in two patients in Italy in 2003 [132]. The second report of TDR-TB came from Iran and was reported by Velayati et al., in 2009 [133]. Subsequently, more cases have also been reported in India and South Africa [132]. The WHO consultative meeting on TDR-TB did not officially endorse the 'TDR-TB' terminology. Some articles suggested the new term 'Extensively-XDR-TB or XXDR-TB' to define TDR-TB strain, because recently, two new drugs, BDQ and DLM, have been approved by the FDA and may offer therapeutic solutions for TDR-TB [132]. Moreover, with newer anti-TB agents in the pipeline, there is hope of identifying drugs that will be effective against both drug-susceptible and drug-resistant MTB and this categorization will soon be obsolete.

\section{Host-directed therapies offer novel treatments for TB}

Considering the restrictions of therapeutic options for the treatment of MDR-TB, the increase in the MDR, XDR and TDR cases worldwide has drawn attention to adjunct host-directed therapies (HDTs) to treat these infections [134]. HDTs, including new and repurposed drugs, biologics, and cellular therapies, have been proposed to shorten treatment duration, prevent resistance, and reduce lung injury, by promoting autophagy, antimicrobial peptide production, other macrophage effector mechanisms, and inhibiting mechanisms causing lung inflammation and matrix destruction [134-136]. It is hoped that combinations of HDTs with anti-TB drug regimens will reduce the duration of therapy, provide better treatment outcomes, reduce the risk 
of developing further drug resistance, and decrease the chances of relapse or reinfection [136].

The Host-Directed Therapies Network (HDT-NET) consortium of 64 partners was launched in April 2015 [74]. This network plans to take forward a wide range of HDTs in randomized, placebo-controlled clinical trials as adjuncts to current TB treatment regimens [136]. Development of HDTs for TB are focused on two general approaches. The first, modulates host inflammatory pathways to reduce aberrant or excessive inflammation and lung tissue destruction while the second increases components of the host's innate and adaptive immune effector mechanisms [137]. It concluded that several drugs already approved for other diseases were ready for clinical assessment in phase IIb/III trials [135]. Examples are as follows: a) Vitamin D induces the expression and release of innate antimicrobial peptides such as cathelicidin, and its effects can be enhanced by combining it with the histone deacetylase inhibitor phenylbutyrate [138]. b) Metformin is a treatment of choice for diabetes. It was identified as an autophagy inducer in a screening of AMP-activated protein kinase activators that inhibited intracellular growth of MTB [139]. c) Several non-steroidal anti-inflammatory drugs (NSAIDs) like aspirin reduce inflammation and tissue pathology, and also have potential to help patients who are co-infected with TB and HIV [137]. d) Doxycycline non-specifically inhibits matrix metalloproteinase (MMPs) at subantimicrobial concentrations. MMPs causes tissue damage through the loss of collagen and other structural proteins; they have been shown in animal models of TB to play an important part in lung destruction [140]. e) Statins such as simvastatin and rosuvastatin have anti-inflammatory effects, and induce autophagy and phagosome maturation [141]. f) Imatinib is a tyrosine kinase inhibitor approved for the treatment of chronic myelogenous leukaemia. In MTB-infected mice and macrophages, low doses of imatinib promoted myelopoiesis, phagosome maturation and acidification and autophagy, thereby reducing bacillary survival [142]. g) CC-11050 inhibits the production of several proinflammatory cytokines (including TNF) by increasing cellular cyclic AMP. In mice and rabbits chronically infected with MTB, CC-11050 reduces the number and size of lung granulomas and accelerates INH-induced bacillary clearance [143].

\section{Conclusion}

Despite the availability of effective antibiotics for TB for the past half-century, it remains an important global health problem; there are $\sim 10$ million active TB cases and $\sim 1.5$ million TB-induced deaths per year. In the last few years, a significant number of new anti-TB drugs entered the preclinical and clinical trials and the mechanism of action of most of them is known. Recently, the FDA allowed the use of BDQ for the treatment of pulmonary TB in adults affected by MDR-MTB strains; finally, after several decades a new compound had been introduced. Improved understanding of the new drug resistance mechanisms in TB will help develop more reliable molecular tests and increase treatment efficacy. However, how drug resistance really develops in patients and the factors that facilitate resistance development are still poorly understood, and merit further study. Furthermore, knowledge of the molecular basis of drug resistance will allow a more rational development of new drugs; something that is urgently needed, when taking into account the increasing rates of MDR- and XDR-TB around the world.

\section{Contributors}

MH, VL and DDS initiated the idea of this study. VL and AZB developed the first and final draft of the manuscript. DDS, MT, LF and MD developed the second draft of the manuscript. All authors reviewed and contributed to revisions and finalized the drafts.

\section{Disclosure of interest}

There are no conflicts of interest.

\section{References}

[1] Jawed Ahsan M, et al. Tuberculosis: current treatment, diagnostics, and newer antitubercular agents in clinical trials. Infect Disord - Drug Targets 2015;15(1):32-41.

[2] Nguyen L. Antibiotic resistance mechanisms in M. tuberculosis: an update. Arch Toxicol 2016;90(7):1585-604.

[3] Zhang Y, Yew W. Mechanisms of drug resistance in Mycobacterium tuberculosis: update 2015. Int J Tubercul Lung Dis 2015;19(11):1276-89.

[4] Organization, W.H. Global tuberculosis report 2016. 2016.

[5] Brouqui P, Quenard F, Drancourt M. Old antibiotics for emerging multidrug-resistant/extensively drug-resistant tuberculosis (MDR/XDR-TB). Int J Antimicrob Agents 2017;49(5):554-7.

[6] Diel R, et al. Costs of tuberculosis disease in the European Union: a systematic analysis and cost calculation. Eur Respir J 2014;43(2):554-65.

[7] Law S, et al. Comparing cost-effectiveness of standardised tuberculosis treatments given varying drug resistance. Eur Respir J 2014;43(2):566-81.

[8] Quenard F, et al. Role of second-line injectable antituberculosis drugs in the treatment of MDR/XDR tuberculosis. Int J Antimicrob Agents 2017;50(2):252-4.

[9] Zhang $\mathrm{Y}$, et al. The catalase-peroxidase gene and isoniazid resistance of Mycobacterium tuberculosis. Nature 1992;358(6387):591-3.

[10] Niki M, et al. A novel mechanism of growth phase-dependent tolerance to isoniazid in mycobacteria. J Biol Chem 2012;287(33):27743-52.

[11] Zhang Y, Yew W. Mechanisms of drug resistance in Mycobacterium tuberculosis [State of the art series. Drug-resistant tuberculosis. Edited by CY. Chiang. Number 1 in the series]. Int J Tubercul Lung Dis 2009;13(11):1320-30.

[12] Hazbón MH, et al. Population genetics study of isoniazid resistance mutations and evolution of multidrug-resistant Mycobacterium tuberculosis. Antimicrob Agents Chemother 2006;50(8):2640-9.

[13] Zhang Y, Telenti A. Genetics of drug resistance in Mycobacterium tuberculosis Molecular genetics of mycobacteria Washington, DC: ASM Press; 2000.

[14] Banerjee A, et al. inhA, a gene encoding a target for isoniazid and ethionamide in Mycobacterium tuberculosis. Science-AAAS-Weekly Paper Edition-including Guide to Scientific Information 1994;263(5144):227-9.

[15] Campbell PJ, et al. Molecular detection of mutations associated with first-and second-line drug resistance compared with conventional drug susceptibility testing of Mycobacterium tuberculosis. Antimicrob Agents Chemother 2011;55(5):2032-41.

[16] Guo H, et al. Molecular characterization of isoniazid-resistant clinical isolates of Mycobacterium tuberculosis from the USA. J Med Microbiol 2006;55(11):1527-31.

[17] Shekar S, et al. Detecting novel genetic variants associated with isoniazid-resistant Mycobacterium tuberculosis. PLos One 2014;9(7):e102383.

[18] Almeida Da Silva PE, Palomino JC. Molecular basis and mechanisms of drug resistance in Mycobacterium tuberculosis: classical and new drugs. J Antimicrob Chemother 2011;66(7):1417-30.

[19] Piccaro G, et al. Rifampin induces hydroxyl radical formation in Mycobacterium tuberculosis. Antimicrob Agents Chemother 2014;58(12):7527-33.

[20] Telenti A, et al. Detection of rifampicin-resistance mutations in Mycobacterium tuberculosis. Lancet 1993;341(8846):647-51.

[21] Jamieson F, et al. Profiling of rpoB mutations and MICs for rifampin and rifabutin in Mycobacterium tuberculosis. J Clin Microbiol 2014;52(6):2157-62.

[22] Takayama K, Kilburn JO. Inhibition of synthesis of arabinogalactan by ethambutol in Mycobacterium smegmatis. Antimicrob Agents Chemother 1989;33(9):1493-9.

[23] Telenti A, et al. The emb operon, a gene cluster of Mycobacterium tuberculosis involved in resistance to ethambutol. Nat Med 1997;3(5):567-70.

[24] Ramaswamy SV, et al. Molecular genetic analysis of nucleotide polymorphisms associated with ethambutol resistance in human isolates ofMycobacterium tuberculosis. Antimicrob Agents Chemother 2000;44(2):326-36.

[25] Sreevatsan S, et al. Ethambutol resistance in Mycobacterium tuberculosis: critical role of embB mutations. Antimicrob Agents Chemother 1997;41(8):1677-81.

[26] Safi H, et al. Transfer of embB codon 306 mutations into clinical Mycobacterium tuberculosis strains alters susceptibility to ethambutol, isoniazid, and rifampin. Antimicrob Agents Chemother 2008;52(6):2027-34.

[27] Alcaide F, Pfyffer GE, Telenti A. Role of embB in natural and acquired resistance to ethambutol in mycobacteria. Antimicrob Agents Chemother 1997:41(10):2270-3.

[28] He L, et al. ubiA (Rv3806c) encoding DPPR synthase involved in cell wall synthesis is associated with ethambutol resistance in Mycobacterium tuberculosis. Tuberculosis 2015;95(2):149-54.

[29] Zhang Y, et al. Mechanisms of pyrazinamide action and resistance. Microbiol Spectr 2013;2(4):1.

[30] Zignol M, et al. Population-based resistance of Mycobacterium tuberculosis isolates to pyrazinamide and fluoroquinolones: results from a multicountry surveillance project. Lancet Infect Dis 2016;16(10):1185-92.

[31] Zhang Y, Mitchison D. The curious characteristics of pyrazinamide: a review. Int J Tubercul Lung Dis 2003;7(1):6-21.

[32] Scorpio A, Zhang Y. Mutations in pncA, a gene encoding pyrazinamidase/nicotinamidase, cause resistance to the antituberculous drug pyrazinamide in tubercle bacillus. Nat Med 1996;2(6):662-7.

[33] Shi W, et al. Pyrazinamide inhibits trans-translation in Mycobacterium tuberculosis. Science 2011;333(6049):1630-2.

[34] Shi W, et al. Aspartate decarboxylase (PanD) as a new target of pyrazinamide in Mycobacterium tuberculosis. Emerg Microb Infect 2014;3(8):e58.

[35] Scorpio A, et al. Characterization of pncA mutations in pyrazinamide-resistant Mycobacterium tuberculosis. Antimicrob Agents Chemother 1997;41(3):540-3. 
[36] Cheng S-J, et al. pncA mutations as a major mechanism of pyrazinamide resistance in Mycobacterium tuberculosis: spread of a monoresistant strain in Quebec, Canada. Antimicrob Agents Chemother 2000;44(3):528-32.

[37] Sreevatsan S, et al. Mutations associated with pyrazinamide resistance in pncA of Mycobacterium tuberculosis complex organisms. Antimicrob Agents Chemother 1997;41(3):636-40.

[38] Hirano K, et al. Mutation in pncA is a major mechanism of pyrazinamide resistance in Mycobacterium tuberculosis. Tuber Lung Dis 1998;78(2):117-22.

[39] Morlock GP, et al. Phenotypic characterization of pncAMutants of Mycobacterium tuberculosis. Antimicrob Agents Chemother 2000;44(9):2291-5.

[40] Feuerriegel S, et al. Mycobacterium canettii is intrinsically resistant to both pyrazinamide and pyrazinoic acid. J Antimicrob Chemother 2013:dkt042.

[41] Finken M, et al. Molecular basis of streptomycin resistance in Mycobacterium tuberculosis: alterations of the ribosomal protein S12 gene and point mutations within a functional 16S ribosomal RNA pseudoknot. Mol Microbiol 1993;9(6):1239-46.

[42] Nair J, et al. The rpsL gene and streptomycin resistance in single and multiple drug-resistant strains of Mycobacterium tuberculosis. Mol Microbiol 1993;10(3):521-7.

[43] Okamoto S, et al. Loss of a conserved 7-methylguanosine modification in 16S rRNA confers low-level streptomycin resistance in bacteria. Mol Microbiol 2007;63(4):1096-106.

[44] Alangaden GJ, et al. Mechanism of resistance to amikacin and kanamycin in Mycobacterium tuberculosis. Antimicrob Agents Chemother 1998;42(5):1295-7.

[45] Zaunbrecher MA, et al. Overexpression of the chromosomally encoded aminoglycoside acetyltransferase eis confers kanamycin resistance in Mycobacterium tuberculosis. Proc Natl Acad Sci Unit States Am 2009;106(47):20004-9.

[46] Maus CE, Plikaytis BB, Shinnick TM. Mutation of tlyA confers capreomycin resistance in Mycobacterium tuberculosis. Antimicrob Agents Chemother 2005;49(2):571-7.

[47] Maus CE, Plikaytis BB, Shinnick TM. Molecular analysis of cross-resistance to capreomycin, kanamycin, amikacin, and viomycin in Mycobacterium tuberculosis. Antimicrob Agents Chemother 2005;49(8):3192-7.

[48] Schoeffler AJ, Berger JM. DNA topoisomerases: harnessing and constraining energy to govern chromosome topology. Q Rev Biophys 2008;41(01):41-101.

[49] Piton J, et al. Structural insights into the quinolone resistance mechanism of Mycobacterium tuberculosis DNA gyrase. PLos One 2010;5(8):e12245.

[50] Vetting MW, et al. Pentapeptide repeat proteins. Biochemistry 2006;45(1):1-10.

[51] Vetting MW, et al. The pentapeptide repeat proteins. Biochemistry 2006;45(1):1-10

[52] Hegde SS, et al. A fluoroquinolone resistance protein from Mycobacterium tuberculosis that mimics DNA. Science 2005;308(5727):1480-3.

[53] Mérens A, et al. The pentapeptide repeat proteins MfpAMt and QnrB4 exhibit opposite effects on DNA gyrase catalytic reactions and on the ternary gyrase-DNAquinolone complex. J Bacteriol 2009;191(5):1587-94.

[54] Zhang Z, et al. Prevalence and molecular characterization of fluoroquinolone-resistant Mycobacterium tuberculosis isolates in China. Antimicrob Agents Chemother 2014;58(1):364-9.

[55] Sirgel FA, et al. gyrA mutations and phenotypic susceptibility levels to ofloxacin and moxifloxacin in clinical isolates of Mycobacterium tuberculosis. J Antimicrob Chemother 2012;67(5):1088-93.

[56] Maruri F, et al. A systematic review of gyrase mutations associated with fluoroquinolone-resistant Mycobacterium tuberculosis and a proposed gyrase numbering system. J Antimicrob Chemother 2012;67(4):819-31.

[57] Long Q, et al. gyrA/B fluoroquinolone resistance allele profiles amongs Mycobacterium tuberculosis isolates from mainland China. Int J Antimicrob Agents 2012;39(6):486-9.

[58] Lu J, et al. Mechanisms of fluoroquinolone monoresistance in Mycobacterium tuberculosis. FEMS Microbiol Lett 2014;353(1):40-8.

[59] DeBarber AE, et al. Ethionamide activation and sensitivity in multidrug-resistan Mycobacterium tuberculosis. Proc Natl Acad Sci Unit States Am 2000;97(17):9677-82.

[60] Baulard AR, et al. Activation of the pro-drug ethionamide is regulated in mycobacteria. J Biol Chem 2000;275(36):28326-31.

[61] Vannelli TA, Dykman A, de Montellano PRO. The antituberculosis drug ethionamide is activated by a flavoprotein monooxygenase. $\mathrm{J}$ Biol Chem 2002;277(15):12824-9.

[62] Strych U, et al. Characterization of the alanine racemases from two mycobacteria. FEMS Microbiol Lett 2001;196(2):93-8.

[63] Caceres NE, et al. Overexpression of the D-alanine racemase gene confers resistance to D-cycloserine in Mycobacterium smegmatis. J Bacteriol 1997;179(16):5046-55.

[64] Feng Z, Barletta RG. Roles of Mycobacterium smegmatis D-alanine: D-alanine ligase and D-alanine racemase in the mechanisms of action of and resistance to the peptidoglycan inhibitor D-cycloserine. Antimicrob Agents Chemother 2003;47(1):283-91.

[65] Prosser GA, de Carvalho LP. Metabolomics reveal d-alanine: d-alanine ligase as the target of d-cycloserine in Mycobacterium tuberculosis. ACS Med Chem Lett 2013;4(12):1233-7.

[66] Chen JM, et al. A point mutation in cycA partially contributes to the D-cycloserine resistance trait of Mycobacterium bovis BCG vaccine strains. PLos One 2012;7(8):e43467.

[67] Rengarajan J, et al. The folate pathway is a target for resistance to the drug paraaminosalicylic acid (PAS) in mycobacteria. Mol Microbiol 2004;53(1):275-82.

[68] Ratledge C. Iron, mycobacteria and tuberculosis. Tuberculosis 2004;84(1):110-30.

[69] Zheng J, et al. para-Aminosalicylic acid is a prodrug targeting dihydrofolate reductase in Mycobacterium tuberculosis. J Biol Chem 2013;288(32):23447-56.

[70] Chakraborty S, et al. Para-aminosalicylic acid acts as an alternative substrate of folate metabolism in Mycobacterium tuberculosis. Science 2013;339(6115):88-91.

[71] Mathys V, et al. Molecular genetics of para-aminosalicylic acid resistance in clinical isolates and spontaneous mutants of Mycobacterium tuberculosis. Antimicrob Agents Chemother 2009;53(5):2100-9.

[72] Zhao F, et al. Binding pocket alterations in dihydrofolate synthase confer resistance to para-aminosalicylic acid in clinical isolates of Mycobacterium tuberculosis. Antimicrob Agents Chemother 2014;58(3):1479-87.

[73] Laughon BE, Nacy CA. Tuberculosis-drugs in the 2016 development pipeline. Nature reviews. Dis Primers 2017;3:17015.

[74] Wallis RS, et al. Tuberculosis_advances in development of new drugs, treatment regimens, host-directed therapies, and biomarkers. Lancet Infect Dis 2016;16(4):e34-46.

[75] Fernandes GFDS, Dos Santos JL. Tuberculosis-current advances in development of new drugs against multidrug-resistant strains. EC Microbiol 2017;6:60-2.

[76] Andries K, et al. A diarylquinoline drug active on the ATP synthase of Mycobacterium tuberculosis. Science 2005;307(5707):223-7.

[77] Mahajan R. Bedaquiline: first FDA-approved tuberculosis drug in 40 years. Int J Appl Basic Med Res 2013:3(1):1-2.

[78] Matteelli A, et al. TMC207: the first compound of a new class of potent anti-tuberculosis drugs. Future Microbiol 2010;5(6):849-58.

[79] Koul A, et al. Diarylquinolines are bactericidal for dormant mycobacteria as a result of disturbed ATP homeostasis. J Biol Chem 2008;283(37):25273-80.

[80] Haagsma AC, et al. Selectivity of TMC207 towards mycobacterial ATP synthase compared with that towards the eukaryotic homologue. Antimicrob Agents Chemother 2009;53(3):1290-2.

[81] Huitric E, et al. In vitro antimycobacterial spectrum of a diarylquinoline ATP synthase inhibitor. Antimicrob Agents Chemother 2007;51(11):4202-4.

[82] Huitric E, et al. Rates and mechanisms of resistance development in Mycobacterium tuberculosis to a novel diarylquinoline ATP synthase inhibitor. Antimicrob Agents Chemother 2010;54(3):1022-8.

[83] Biuković G, et al. Variations of subunit $\varepsilon$ of the Mycobacterium tuberculosis F1Fo ATP synthase and a novel model for mechanism of action of the tuberculosis drug TMC207. Antimicrob Agents Chemother 2013;57(1):168-76.

[84] Hartkoorn RC, Uplekar S, Cole ST. Cross-resistance between clofazimine and bedaquiline through upregulation of MmpL5 in Mycobacterium tuberculosis. Antimicrob Agents Chemother 2014;58(5):2979-81.

[85] Andries K, et al. Acquired resistance of Mycobacterium tuberculosis to bedaquiline. PLos One 2014;9(7):e102135.

[86] Cox E, Laessig K. FDA approval of bedaquiline - the benefit-risk balance for drugresistant tuberculosis. N Engl J Med 2014;371(8):689-91.

[87] Deoghare S. Bedaquiline: a new drug approved for treatment of multidrug-resistant tuberculosis. Indian J Pharmacol 2013;45(5):536.

[88] Diacon AH, et al. Multidrug-resistant tuberculosis and culture conversion with bedaquiline. N Engl J Med 2014;371(8):723-32.

[89] Pontali E, et al. Bedaquiline and multidrug-resistant tuberculosis: a systematic and critical analysis of the evidence. Eur Respir J 2016;47(2):394-402.

[90] Diacon AH, et al. The diarylquinoline TMC207 for multidrug-resistant tuberculosis. N Engl J Med 2009;360(23):2397-405.

[91] Provisional CDC guidelines for the use and safety monitoring of bedaquiline fumarate (Sirturo) for the treatment of multidrug-resistant tuberculosis. MMWR Recomm Rep 2013;62(Rr-09):1-12.

[92] Field SK. Bedaquiline for the treatment of multidrug-resistant tuberculosis: great promise or disappointment? Therap Adv Chronic Dis 2015;6(4):170-84.

[93] Stover CK, et al. A small-molecule nitroimidazopyran drug candidate for the treatment of tuberculosis. Nature 2000;405(6789):962-6.

[94] Singh R, et al. PA-824 kills nonreplicating Mycobacterium tuberculosis by intracellular NO release. Science 2008;322(5906):1392-5.

[95] Manjunatha UH, et al. Identification of a nitroimidazo-oxazine-specific protein involved in PA-824 resistance in Mycobacterium tuberculosis. Proceed Natnl Acad Sci U S A 2006;103(2):431-6.

[96] Feuerriegel S, et al. Impact of fgd1 and ddn diversity in Mycobacterium tuberculosis complex on in vitro susceptibility to PA-824. Antimicrob Agents Chemother 2011;55(12):5718-22.

[97] Li SY, et al. Bactericidal and Sterilizing Activity of a Novel Regimen with Bedaquiline, Pretomanid, Moxifloxacin, and Pyrazinamide in a Murine Model of Tuberculosis. Antimicrob Agents Chemother 2017;61(9).

[98] Matsumoto M, et al. OPC-67683, a nitro-dihydro-imidazooxazole derivative with promising action against tuberculosis in vitro and in mice. PLoS Med 2006;3(11):e466.

[99] Schena E, et al. Delamanid susceptibility testing of Mycobacterium tuberculosis using the resazurin microtitre assay and the BACTEC ${ }^{\mathrm{TM}}$ MGIT ${ }^{\mathrm{TM}} 960$ system. J Antimicrob Chemother 2016;71(6):1532-9.

[100] Barry P, O'Connor T. Novel agents in the management of Mycobacterium tuberculosis disease. Curr Med Chem 2007;14(18):2000-8.

[101] Gler MT, et al. Delamanid for multidrug-resistant pulmonary tuberculosis. N Engl J Med 2012;366(23):2151-60.

[102] Saliu OY, et al. Bactericidal activity of OPC-67683 against drug-tolerant Mycobacterium tuberculosis. J Antimicrob Chemother 2007;60(5):994-8.

[103] Organization, W.H. The use of delamanid in the treatment of multidrug-resistant tuberculosis: interim policy guidance. 2014

[104] Skripconoka V, et al. Delamanid improves outcomes and reduces mortality in multidrug-resistant tuberculosis. Eur Respir J 2013;41(6):1393-400.

[105] Wells CD, et al. Long-term mortality assessment of multidrug-resistant tuberculosis patients treated with delamanid. Eur Respir J 2015;45(5):1498-501. 
[106] Choi K-P, Kendrick N, Daniels L. Demonstration that fbiC is required by Mycobacterium bovis BCG for coenzyme F420 and FO biosynthesis. J Bacteriol 2002;184(9):2420-8.

[107] Forouhar F, et al. Molecular insights into the biosynthesis of the F420 coenzyme. J Biol Chem 2008;283(17):11832-40.

[108] Xavier AS, Lakshmanan M. Delamanid: A new armor in combating drug-resistant tuberculosis. J Pharmacol Pharmacother 2014;5(3):222-4.

[109] Hafkin J, Frias M, Hesseling A. Pharmacokinetics and safety of delamanid in pediatric MDR-TB patients, ages 6-17 years. Interscience conference on antimicrobial agents and chemotherapy.[poster A-960]. San Diego, CA, USA: ICAAC; 2015.

[110] Hafkin J, Gler M, Frias M. Long-term safety, tolerability, and pharmacokinetics of delamanid in children aged 12-17. The 46th union worls conference on lung health, cape Town, SA. 2015.

[111] Tadolini M, et al. Compassionate use of new drugs in children and adolescents with multidrug-resistant and extensively drug-resistant tuberculosis: early experiences and challenges. Eur Respir J 2016;48(3):938-43.

[112] Tadolini M, et al. First case of extensively drug-resistant tuberculosis treated with both delamanid and bedaquiline. Eur Respir J 2016;48(3):935-8.

[113] Diacon A, et al. Early bactericidal activity of delamanid (OPC-67683) in smearpositive pulmonary tuberculosis patients. Int J Tubercul Lung Dis 2011;15(7):949-54.

[114] Jindani A, et al. The Early Bactericidal Activity of Drugs in Patients with Pulmonary Tuberculosis 1, 2. Am Rev Respirat Dis 1980;121(6):939-49.

[115] Bialvaei AZ, et al. Linezolid: a promising option in the treatment of Gram-positives. J Antimicrob Chemother 2016:dkw450.

[116] Barbachyn MR, et al. Identification of a novel oxazolidinone (U-100480) with potent antimycobacterial activity. J Med Chem 1996;39(3):680-5.

[117] Barrett J. Linezolid Pharmacia Corp. Curr Opin Invest Drugs 2000;1(2):181-7.

[118] McKee E, et al. Inhibition of mammalian mitochondrial protein synthesis by oxazolidinones. Antimicrob Agents Chemother 2006;50(6):2042-9.

[119] Lee M, et al. Linezolid for treatment of chronic extensively drug-resistant tuberculosis. N Engl J Med 2012;367(16):1508-18.

[120] Hillemann D, Rüsch-Gerdes S, Richter E. In vitro-selected linezolid-resistant Mycobacterium tuberculosis mutants. Antimicrob Agents Chemother 2008;52(2):800-1.

[121] Beckert P, et al. rplC T460C identified as a dominant mutation in linezolid-resistant Mycobacterium tuberculosis strains. Antimicrob Agents Chemother 2012;56(5):2743-5.

[122] Zhang Z, et al. Beijing genotype of Mycobacterium tuberculosis is significantly associated with linezolid resistance in multidrug-resistant and extensively drugresistant tuberculosis in China. Int J Antimicrob Agents 2014;43(3):231-5.

[123] Cynamon M, et al. Activities of Several Novel Oxazolidinones againstMycobacterium tuberculosis in a Murine Model. Antimicrob Agents Chemother 1999;43(5):1189-91.

[124] Shaw KJ, Barbachyn MR. The oxazolidinones: past, present, and future. Ann N Y Acad Sci 2011;1241(1):48-70.

[125] Wallis RS, et al. Biomarker-assisted dose selection for safety and efficacy in early development of PNU-100480 for tuberculosis. Antimicrob Agents Chemother 2011;55(2):567-74.

[126] Williams KN, et al. Addition of PNU-100480 to first-line drugs shortens the time needed to cure murine tuberculosis. Am J Respir Crit Care Med 2009;180(4):371-6.

[127] Reddy VM, et al. SQ109 and PNU-100480 interact to kill Mycobacterium tuberculosis in vitro. J Antimicrob Chemother 2012:dkr589.

[128] Wallis RS, et al. Rapid evaluation in whole blood culture of regimens for XDR-TB containing PNU-100480 (sutezolid), TMC207, PA-824, SQ109, and pyrazinamide. PLos One 2012;7(1):e30479.

[129] Pethe K, et al. Discovery of Q203, a potent clinical candidate for the treatment of tuberculosis. Nat Med 2013;19(9):1157-60.

[130] Hoagland DT, et al. New agents for the treatment of drug-resistant Mycobacterium tuberculosis. Adv Drug Deliv Rev 2016;102:55-72.

[131] Udwadia ZF, et al. Totally drug-resistant tuberculosis in India. Clin Infect Dis 2012;54(4):579-81.

[132] Parida S, et al. Totally drug-resistant tuberculosis and adjunct therapies. J Intern Med 2015;277(4):388-405.

[133] Velayati AA, et al. Emergence of new forms of totally drug-resistant tuberculosis bacilli: super extensively drug-resistant tuberculosis or totally drug-resistant strains in Iran. CGST J 2009;136(2):420-5.

[134] Zumla A, et al. Host-directed therapies for tackling multi-drug resistant tuberculosis: learning from the Pasteur-Bechamp debates. Clin Infect Dis 2015;61(9):1432-8.

[135] Zumla A, Maeurer M. Host-directed therapies for multidrug resistant tuberculosis. Int J Mycobacteriol 2016;5:S21-2.

[136] Wallis RS, Hafner R. Advancing host-directed therapy for tuberculosis. Nat Rev Immunol 2015;15(4):255-63.

[137] Zumla A, et al. Towards host-directed therapies for tuberculosis [Comment]. Nat Rev Drug Discov 2016;14(8):511-2.

[138] Machelart A, et al. Host-directed therapies offer novel opportunities for the fight against tuberculosis. Drug Discov Today 2017.

[139] Singhal A, et al. Metformin as adjunct antituberculosis therapy. Sci Transl Med 2014;6(263). 263ra159-263ra159.

[140] Ong CW, et al. Neutrophil-derived MMP-8 drives AMPK-dependent matrix destruction in human pulmonary tuberculosis. PLoS Pathog 2015;11(5):e1004917.

[141] Parihar SP, et al. Statin therapy reduces the mycobacterium tuberculosis burden in human macrophages and in mice by enhancing autophagy and phagosome maturation. J Infect Dis 2013;209(5):754-63.

[142] Napier RJ, et al. Imatinib-sensitive tyrosine kinases regulate mycobacterial pathogenesis and represent therapeutic targets against tuberculosis. Cell Host Microbe 2011;10(5):475-85.

[143] Paul C, et al. Efficacy and safety of apremilast, an oral phosphodiesterase 4 inhibitor, in patients with moderate-to-severe plaque psoriasis over 52 weeks: a phase III, randomized controlled trial (ESTEEM 2). Br J Dermatol 2015;173(6):1387-99. 